\title{
Criando elos
}

Luís Miguel Monteiro, ${ }^{*}$ Cláudio Carril,* Vera Santos, ${ }^{* *}$ Sergey Borisushkin, ${ }^{* * *}$ Carla Correia, ${ }^{* * * *}$

Cláudia Ferreira, ${ }^{* * * *}$ Helena Santos, ${ }^{* * * * *}$ Helena Sequeira, ${ }^{* * * * * *}$ Luísa Simão, ${ }^{* * * * * * *}$ Luísa Veiga********

\section{RESUMO}

Introdução: Esta publicação pretende divulgar uma metodologia de articulação inter-institucional.

Desde 2000, equipas do Centro de Saúde de Tábua e do Departamento de Pedopsiquiatria do Hospital Pediátrico (Centro Hospitalar de Coimbra) reúnem periodicamente com vista à triagem, discussão e supervisão de casos clínicos com problemáticas na área da Psiquiatria da Infância e Adolescência.

Após sete anos de trabalho conjunto, é feita reavaliação desta parceria complementada com a análise estatística de 2007.

Objectivos: Melhorar a acessibilidade, triar e supervisionar casos clínicos, promover a circulação bidireccional de informação clínica, rentabilizar recursos e promover a formação de médicos de família e psicólogos.

Metodologia: Reuniões interdisciplinares presenciais mensais; contacto escrito e telefónico.

Resultados: Realizaram-se oito reuniões/ano; foram discutidos em média 3,75 primeiros casos/reunião; dos 30 novos casos discutidos em 2007, 40\% necessitaram de consulta no Departamento de Pedopsiquiatria; 60\% necessitaram apenas de intervenção do Centro de Saúde; lista de espera de cinco semanas (média); motivos mais frequentes de discussão: problemas de comportamento e aprendizagem, disfuncionalidade familiar inerente a psicopatologia familiar e troca de informações bilateral.

Discussão: Aumento de primeiros casos, devido provavelmente à integração de um novo médico de família na consultadoria; decréscimo de primeiros casos orientados para o Departamento devido à boa qualidade do trabalho desenvolvido nos Cuidados Primários; motivos de referenciação/discussão entre o médico de família e o psiquiatra em concordância com a bibliografia consultada.

Conclusões: com esta parceria, existe maior participação e mobilização dos recursos locais, menor necessidade de deslocação das famílias para consultas no Departamento de Pedopsiquiatria, diminuição das listas de espera e altas mais precoces. Apenas não alcançámos o objectivo dos estágios de formação, por falta de técnicos locais (nomeadamente psicólogos). Existem poucas equipas a trabalhar em consultadoria. Sugere-se maior implementação deste modelo organizacional que fortaleça a ligação entre Cuidados de Saúde Primários e Secundários.

Palavras-chave: Consultadoria; Medicina Geral e Familiar; Psiquiatria da Infância e Adolescência.

\section{INTRODUÇÃO}

A consultadoria em Psiquiatria da Infância e Adolescência é uma forma de Pedopsiquiatria de ligação que permite a abordagem dos problemas de saúde mental de um modo mais eficaz. ${ }^{1}$

Esta metodologia está de acordo com os valores, princípios e objectivos estabelecidos no Plano Nacional de Saúde Mental 2007-2016. Neste plano, é preco-

\footnotetext{
*Interno do $2^{\circ}$ ano de Medicina Geral e Familiar do Centro de Saúde de Tábua **Interna do $5^{\circ}$ ano de Psiquiatria da Infância e Adolescência do Departamento de Pedopsiquiatria do HP - CHC

***Interno do $3^{\circ}$ ano de Medicina Geral e Familiar do Centro de Saúde de Tábua ****Assistente de Medicina Geral e Familiar do Centro de Saúde de Tábua *****Assessora de Serviço Social do Departamento de Pedopsiquiatria do HP _- CHC ******Chefe de Serviço de Medicina Geral e Familiar do Centro de Saúde de Tábua *******Técnica Superior Principal de Psicologia Clínica do Departamento de Pedopsiquiatria do HP - CHC

********Assistente Hospitalar Graduada de Psiquiatria da Infância e Adolescência do Departamento de Pedopsiquiatria do HP - CHC
}

nizado que a prestação de cuidados na área da saúde mental da infância e adolescência, a nível dos Cuidados de Saúde Primários, «deve ser assegurada por Grupos ou Núcleos de Apoio à Saúde Mental Infantil, constituídos por profissionais dos centros de saúde e unidades de saúde familiar, com a consultoria de um Psiquiatra da Infância e Adolescência do serviço especializado local.»²

Inicialmente, a equipa de Tábua integrava médicos de família do Centro de Saúde, psicóloga, e assistente social das estruturas locais (Câmara, Misericórdia, Centro de Acolhimento Temporário). Posteriormente, após remodelações internas destas instituições, passou a ser constituída essencialmente por médicos deste Centro de Saúde e pontualmente outros profissionais ligados aos casos, nomeadamente assistente social.

Esta consultadoria foi iniciada no ano 2000 e consiste numa articulação inter-institucional, actualmente 
entre a equipa do Centro de Saúde de Tábua e uma equipa do Departamento de Pedopsiquiatria do Hospital Pediátrico - Centro Hospitalar de Coimbra (HP-CHC) (Psiquiatra da Infância e Adolescência, internos da especialidade, psicóloga, assistente social e eventualmente estagiários destas áreas).

Anteriormente à implementação da consultadoria, existia apenas uma informação de retorno esporádica (por carta ou via telefónica) para os médicos de família, relativamente às crianças seguidas em cuidados secundários. Por outro lado, a Pedopsiquiatria não tinha conhecimento da realidade das situações e dos recursos existentes a nível local.

Este trabalho em rede proporciona uma maior qualidade e rentabilização dos serviços e fomenta a sensibilização e melhor avaliação dos problemas de saúde mental infantil e juvenil pelos profissionais dos centros de saúde. ${ }^{1-3}$

Neste artigo, descrevem-se os objectivos e as metodologias utilizadas nesta consultadoria e faz-se uma actualização estatística relativa a 2007, analisando-se: os principais motivos de observação, o número de casos discutidos e as orientações preconizadas, bem como o número de casos que necessitaram de observação no Departamento e o respectivo tempo de espera. Conclui-se com as vantagens que este tipo de trabalho nos parece ter.

\section{OBJECTIVOS}

Foi elaborado um protocolo com os seguintes objectivos:

1) Melhorar a acessibilidade e resposta, em tempo útil, às necessidades das crianças e jovens com sintomatologia psiquiátrica.

2) Triar e supervisionar casos clínicos.

3) Promover a circulação de informação clínica bidireccional.

4) Rentabilizar recursos e mobilizar as redes de suporte locais.

5) Formar médicos de família e psicólogos (supervisão, estágios de formação e participação em acções de formação organizadas pelo Centro de Saúde).

\section{METODOLOGIA}

As reuniões interdisciplinares desta consultadoria ocorrem mensalmente (com duração de cerca de quatro ho- ras) no Departamento de Pedopsiquiatria do HP-CHC, para supervisão de casos clínicos, com vista à sua triagem e orientação terapêutica (sempre que possível local).

Quando necessário, a interligação é feita também por escrito ou por contacto telefónico para troca de informações e orientações terapêuticas.

Estas reuniões têm como ordem de trabalhos:

a) Informações gerais relativas a mudanças de metodologia organizacional, legislação e às redes de apoio social (Instituições Particulares de Solidariedade Social - IPSS; Comissão de Protecção de Crianças e Jovens - CPCJ) e projectos existentes (Projecto Integrado de Intervenção Precoce - PIIP).

b) Apresentação de casos clínicos seleccionados pelo Centro de Saúde, com discussão entre todos os técnicos da equipa e ponderação das estratégias/intervenções terapêuticas mais indicadas a cada caso.

c) Selecção dos casos que necessitam de observação no Departamento, pela Pedopsiquiatria, Psicologia e/ou Serviço Social (apenas quando os recursos locais não têm competência específica ou são inexistentes).

d) Seguimento dos casos previamente discutidos, mantendo-se a supervisão mesmo após a alta do Departamento.

e) Informação de retorno à equipa do Centro de Saúde após primeiras consultas, avaliações na urgência ou seguimentos por outros pedopsiquiatras.

f) Informações pertinentes acerca de enquadramentos sócio-familiares.

g) Reanálise das orientações (seguimento psicoterapêutico, psicofarmacologia, altas com orientação).

h) Orientações específicas para a Medicina Geral e Familiar, nomeadamente:

1) Informações acerca da dinâmica ou psicopatologia familiar.

2) Desmistificação e sensibilização para acompanhamento psiquiátrico.

3) Orientação dos pais para consulta de Psiquiatria Geral.

4) Análise funcional de comportamentos problemáticos.

5) Informação da psicofarmacologia e/ou pedido de exames complementares de diagnóstico.

6) Avaliação psicológica e/ou social local. 
7) Sinalização para a CPCJ ou Tribunais.

8) Mobilização de outros recursos locais, tais como: contacto com escolas, integração de crianças em infantários, instituições, actividades extracurriculares, projectos da comunidade (análise das redes a mobilizar para a sua implementação).

\section{RESULTADOS}

Desde o início, o número de reuniões de consultadoria/ano foi em média de oito, contemplando interregnos devidos a períodos de férias.

Desde 2000, o número de casos discutidos por reunião foi de um mínimo de 3 a um máximo de 15, muitos já conhecidos, com uma média de primeiros casos, de 1 a 2 por reunião, tendo sido de 3,75 em 2007.

Neste último ano, foram avaliados 48 casos clínicos entre primeiros e seguimentos. Como cada caso pode ser analisado em diversas reuniões ao longo do ano, estes casos corresponderam a 77 discussões.

Na Figura 1 é apresentado o número de primeiros casos/ano. A variação foi de 11 a 30 casos clínicos, sendo este último valor respeitante a 2007.

Relativamente ao sexo, dos 48 casos de 2007, 25 foram do sexo masculino e 23 do feminino. Quanto aos 30 novos casos, predominou o género feminino (16 raparigas / 14 rapazes).

Entre 2000 e 2006 o número de primeiros casos foi de 111, sendo que 59 foram orientados para o Departamento. Os restantes 52 não necessitaram desta orientação.

Do total de primeiros casos de 2007 ( $\mathrm{n}=30$ ), foram orientados para o Departamento apenas 12 (40\%) (Figura 2). Destes, 8 foram observados pela Pedopsiquiatria (foram propostos 10, mas 2 faltaram), 5 foram também avaliados pela Psicologia ( 1 apenas por esta valên-

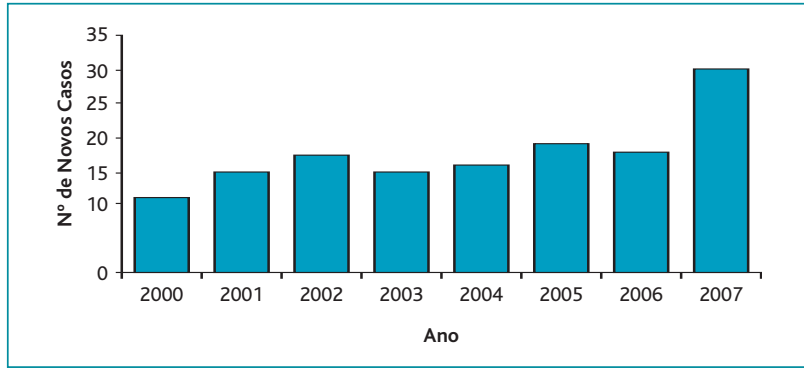

Figura 1. Número de Primeiros Casos em Consultadoria/Ano. cia) e 6 pelo Serviço Social (1 apenas por este serviço). Os restantes 18 (60\%) necessitaram apenas de manter o acompanhamento pelo seu médico de família no Centro de Saúde de Tábua.

O tempo de espera para observação em consulta médica no Departamento foi em média de cinco semanas.

Os motivos mais frequentes de discussão e/ou referenciação, considerando-se a co-morbilidade, foram sobretudo problemas de comportamento e aprendizagem, disfuncionalidade e psicopatologia familiar, bem como troca de informações bilateral, como se pode observar no Quadro I.

\section{DISCUSSÃO}

Analisando a Figura 1 (número de primeiros casos em consultadoria/ano), verificamos que a variação foi de 11 a 18 casos entre 2000 e 2006. Em 2007 foram referenciados 30 primeiros casos. Pensamos que este aumento se deveu provavelmente, ao facto de um novo médico de família ter integrado recentemente a consultadoria, tendo trazido vários casos do seu ficheiro.

Relativamente aos 30 casos avaliados pela primeira vez, curiosamente predominou ligeiramente o género feminino (16 raparigas, 14 rapazes). Dado isto não ser o habitual em Psiquiatria da Infância e Adolescência, ${ }^{4-5-6}$ seria interessante estudar o motivo de referenciação e as idades correspondentes em futuros traba-

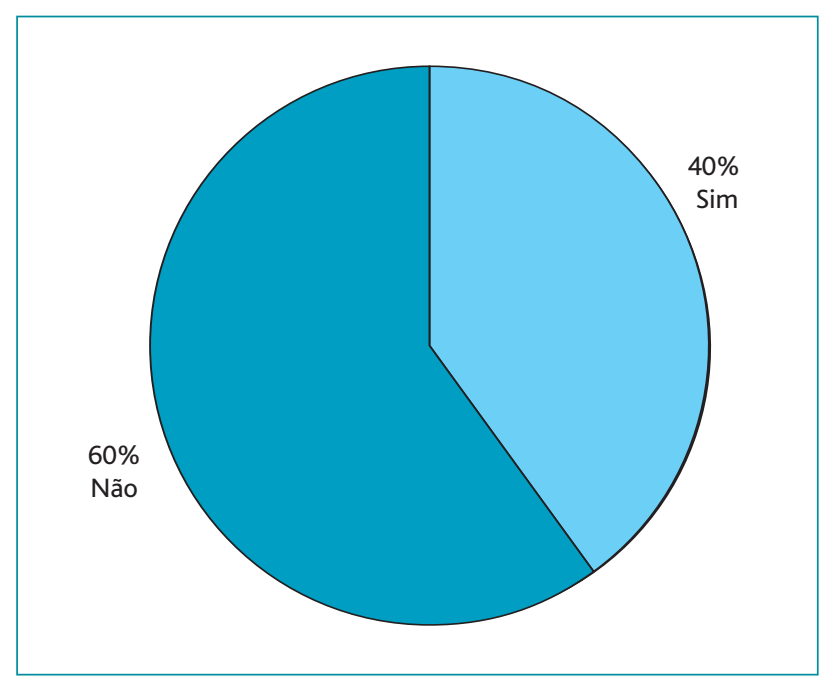

Figura 2. Casos Orientados para o Departamento de Pedopsiquiatria em 2007 
QUADRO I. Motivos de Discussão/Referenciação

Médico de Família $\rightarrow$ Psiquiatra

Motivos de Discussão/Referenciação

Médico de Família $\rightarrow$ Psiquiatra

Problemas de comportamento

Patologia materna

Informação de retorno da consulta

de Pedopsiquiatria

Dificuldades de aprendizagem $\quad 8$

\begin{tabular}{l|l}
\hline Faltas às consultas & 4 \\
\hline
\end{tabular}

\begin{tabular}{ll}
\hline Informação de retorno da & 4
\end{tabular}

Medicina Geral e Familiar

Pedido de informação ao Departamento 3

Encoprese 3

\begin{tabular}{l|l}
\hline Patologia na família alargada & 3 \\
\hline
\end{tabular}

\begin{tabular}{ll}
\hline Somatização & 3
\end{tabular}

\begin{tabular}{ll}
\hline Processo disciplinar escolar & 3
\end{tabular}

Suspeita de abuso/maus tratos 2

\begin{tabular}{ll}
\hline Depressão & 2 \\
\hline
\end{tabular}

\begin{tabular}{ll}
\hline Ansiedade & 2 \\
\hline
\end{tabular}

\begin{tabular}{l|l}
\hline Patologia paterna & 2 \\
\hline
\end{tabular}

\begin{tabular}{l|l}
\hline Luto & 2 \\
\hline
\end{tabular}

Dificuldades relacionamento

professor/aluno

Hiperactividade

\begin{tabular}{ll}
\hline Informação de retorno da avaliação & 1
\end{tabular}

psicológica

\begin{tabular}{l|l}
\hline Psicose & 1 \\
\hline Obsessões & 1 \\
\hline Patologia fraterna & 1
\end{tabular}

lhos, atendendo ao facto da psicopatologia ser distinta consoante o sexo.

Relativamente à Figura 2 (casos orientados para o Departamento em 2007), constata-se que neste ano, não obstante ter havido um aumento de primeiros casos em consultadoria, houve um decréscimo na necessidade de vinda das famílias ao Departamento.

Dado que um trabalho anterior (XVII Encontro Nacional de Psiquiatria da Infância e Adolescência, 2006) revelou que $53 \%$, de um universo de 111 casos, necessitaram de ter consulta da especialidade, ${ }^{7}$ parece de salientar a descida para $40 \%$, tanto mais que corresponde ao dobro do número de primeiros casos, comparativamente a anos anteriores, que tiveram uma média de 15 primeiros casos/ano.
Visto que as situações clínicas não são menos complexas, esta situação parece dever-se a uma boa prise en charge e bom trabalho dos Cuidados Primários.

O tempo de espera para observação em consulta médica no Departamento de Pedopsiquiatria (cinco semanas, em média), foi claramente inferior à lista de espera deste Departamento (quatro meses, no período estudado entre Julho 2006 e Junho de 2007), o que torna também esta metodologia de trabalho muito vantajosa.

Quando consideramos os dados do Quadro I (motivos de discussão/referenciação entre o médico de família e o psiquiatra), verificamos que existe concordância com a bibliografia consultada. ${ }^{5-6,8}$

Garralda refere que a maioria das consultas em Cuidados Primários são motivadas por queixas físicas, não sendo de descurar aquelas cujo motivo é de cariz psicológico ou relativo ao desenvolvimento. ${ }^{4}$ Para estas, os médicos de família têm também que dar resposta, quer ao nível dos Cuidados Primários, quer através da referenciação para um serviço mais especializado (Pedopsiquiatria). A maior parte dos pedidos de consulta pedopsiquiátrica provêm da referenciação da Medicina Familiar.

Vários estudos referem que 2 a 5\% das crianças e jovens que recorrem aos Cuidados de Saúde (Primários ou serviços de Pediatria) apresentam problemas psicológicos. ${ }^{5-6}$ Das crianças que recorrem aos Cuidados de Saúde Primários com distúrbios psiquiátricos, a maioria apresenta queixas somáticas.

\section{CONCLUSÕES}

Segundo Garralda, estima-se que os médicos de família ocupam uma quarta parte do seu tempo com crianças (sobretudo as pré-escolares). ${ }^{4}$ Assim, o contacto com os Cuidados de Saúde Primários é uma experiência comum na infância.

Dados estatísticos do Centro de Saúde de Tábua, referentes a 2007, indicam que, de um total de 45.338 consultas efectuadas, 4.950 são referentes a crianças e jovens dos 0 aos 18 anos, correspondendo a 10,91\%. Este facto revela a importância da existência deste trabalho conjunto entre o Centro de Saúde de Tábua e o Departamento de Pedopsiquiatria do HP - CHC.

Resultados de trabalhos anteriores revelaram que, com esta parceria, existe maior participação e mobilização dos recursos locais, uma menor necessidade de 
deslocação das famílias para consultas no Departamento de Pedopsiquiatria, diminuição das listas de espera e altas mais precoces, ${ }^{7}$ que dados de 2007 continuam a confirmar.

Sabemos que o diagnóstico dos distúrbios psiquiátricos na infância e adolescência efectuado pelos médicos de família é muito reduzido, ${ }^{4,5}$ constatando-se que esse reconhecimento diagnóstico é maior nas crianças com patologia mais grave, daí o interesse da formação contínua que esta consultadoria permite.

Gask et al, em 1997, resumiram os objectivos que se pretendem alcançar com a ligação entre a Psiquiatria e os Cuidados de Saúde Primários: ${ }^{1}$

a) Ajudar os médicos de família a identificar a patologia psiquiátrica.

b) Ajudar os médicos de família a lidar directamente com os casos, dentro das suas competências.

c) Ajudar a definir qual o momento certo para referenciar à Pedopsiquiatria.

d) Partilhar com a equipa dos Cuidados de Saúde Primários a continuidade dos cuidados dos doentes crónicos e permitir explorar e melhorar a relação médico-família bem como a relação entre o médico de família e o especialista de Pedopsiquiatria.

Sete anos depois, entendemos que os objectivos preconizados por Gask, em 1997, foram sobejamente alcançados com esta consultadoria.

Relativamente ao que nos propusemos, apenas não alcançámos o objectivo dos estágios de formação, por falta de técnicos locais (nomeadamente psicólogos). Contudo, também mantivemos a formação contínua bilateral, tendo sido promovidas acções de formação em Encontros locais.

Existem poucas equipas a trabalhar em consultadoria. Sugere-se uma maior implementação deste modelo organizacional que fortaleça a ligação entre Cuidados de Saúde Primários e Secundários.

\section{AGRADECIMENTOS}

Um agradecimento, pela prestimosa colaboração, às enfermeiras Lurdes Patrício e Teresa Leonor do Departamento de Pedopsiquiatria, bem como à Dra. Sara Pedroso, interna de Pedopsiquiatria.

\section{REFERÊNCIAS BIBLIOGRÁFICAS}

1. Gask L, Sibbald B, Creed F. Evaluating models of working at the interface between mental health services and primary care. Br J Psychiatry 1997 Jan; 170: 6-11.

2. Ministério da Saúde, Alto Comissariado da Saúde, Coordenação Nacional para a Saúde Mental. Plano Nacional de Saúde Mental 20072010 - Resumo Executivo. Lisboa. Ministério da Saúde; 2008. p. 3-17, 24-25.

3. Castell R, Biener A, Artner K, Kleeberger E. The treatment of children with psychiatric disorders by general practitioners and paediatricians in South-East Bavaria (author's translation). Monatsschr Kinderheilkd 1980 Sep; 128 (9): 602-5.

4. Garralda ME. Primary Health Care Psychiatry. In: Rutter M, Taylor EA, editors. Child and Adolescent Psychiatry. 4th ed. Oxford: Blackwell; 2005. p. 1090-1100.

5. Garralda ME, Bailey D. Children with psychiatric disorders in primary care. J Child Psychol Psychiatry 1986 Sep; 27 (5): 611-24.

6. Kramer T, Garralda ME. Psychiatric disorders in adolescents in primary care. Br J Psychiatry 1998 Dec; 173: 508-13.

7. Duarte A, Santos H, Sequeira H, Simão L, Veiga L, Neves N, et al. A Pedopsiquiatria sai à rua cá dentro [poster]. XVII Encontro Nacional de Psiquiatria da Infância e Adolescência. 2006.

8. Costello EJ, Edelbrock CS, Costello AJ. Validity of the NIMH Diagnostic Interview Schedule for Children: a comparison between psychiatric and pediatric referrals. J Abnormal Child Psychol 1985 Dec;13(4):579-95.

Os autores declararam não possuir conflitos de interesses

\section{ENDEREÇO PARA CORRESPONDÊNCIA}

Luis Miguel Monteiro

Centro de Saúde de Tábua

Avenida de Coimbra

Apartado 147

3424-909 Tábua

Tlm.: 964622905

E-mail: Imomr45563@gmail.com

Recebido em 23/09/2008

Aceite para publicação em 01/07/2009 


\section{ABSTRACT}

\section{ESTABLISHING CONNECTIONS}

Introduction: This publication aims at showing a methodology of inter-institutional articulation.

Since 2000, teams of the Health Centre of Tábua and of the Department of Child and Adolescence Psychiatry in the Paediatrics' Hospital (Hospital Centre of Coimbra) meet periodically with the objective to screen, discuss and supervise clinical cases related to problems of Child and Adolescence Psychiatry.

After seven years of team work, we have made a reassessment of this partnership, supported by statistical analysis 2007.

Objectives: To improve access, to screen and supervise clinical cases, to promote the bidirectional circulation of clinical data, take advantage of resources and to promote the training of family physicians and psychologists.

Methodology: Monthly actual meetings between the two teams; written and phone contacts.

Results: we have made eight meetings/year; about 3,75 first cases were discussed in each meeting; of the 30 new discussed in 2007, 40\% needed an appointment in the Department of Child and Adolescence Psychiatry; 60\% just needed intervention of the Health Centre; waiting list for an appointment of five weeks (average); the most frequent causes of discussion and/or reference were: behaviour and learning problems, family dysfunction related to family psychopathology and exchange of bilateral data.

Discussion: increase of first cases probably owing to the participation of a new family physician in the meetings; decrease of first cases directed to the Department due to the good quality of the work developed in the Primary Care; causes of reference/discussion between the family physician and the psychiatrist according to the consulted bibliography.

Conclusions: with this partnership, there is an increasing participation and mobilization of the local resources, a decreasing need to lead families to appointments in the try, a decrease of the waiting

Conclusions: with this partnership, there is an increasing participation and mobilization of the local resources, a decreasing need to lead families to appointments in the Department of Child and Adolescence Psychiatry, a decrease of the waiting lists and earlier discharge of the patients. The only objective we did not achieve was the training, due to lack of local technicians (namely psychologists). There are few known partnerships working in this kind of practice. We suggest a stronger implementation of this organizational model to strength the link between Primary and Secondary Health Care.

Keywords: Consultation; General and Family Medicine; Child and Adolescence Psychiatry. 